\title{
GROWTH PROPERTIES AND SEQUENCES OF ZEROS OF ANALYTIC FUNCTIONS IN SPACES OF DIRICHLET TYPE
}

\author{
DANIEL GIRELA ${ }^{\varpi}$ and JOSÉ ÁNGEL PELÁEZ
}

(Received 1 June 2004; revised 3 April 2005)

Communicated by P. C. Fenton

\begin{abstract}
For $0<p<\infty$, we let $\mathscr{D}_{p-1}^{p}$ denote the space of those functions $f$ that are analytic in the unit disc $\Delta=\{z \in \mathbb{C}:|z|<1\}$ and satisfy $\int_{\Delta}(1-|z|)^{p-1}\left|f^{\prime}(z)\right|^{p} d x d y<\infty$. The spaces $\mathscr{D}_{p-1}^{p}$ are closely related to Hardy spaces. We have, $\mathscr{D}_{p-1}^{p} \subset H^{p}$, if $0<p \leq 2$, and $H^{p} \subset \mathscr{D}_{p-1}^{p}$, if $2 \leq p<\infty$. In this paper we obtain a number of results about the Taylor coefficients of $\mathscr{D}_{p-1}^{p}$-functions and sharp estimates on the growth of the integral means and the radial growth of these functions as well as information on their zero sets.
\end{abstract}

2000 Mathematics subject classification: primary 30D35, 30D55, 46E15.

Keywords and phrases: Spaces of Dirichlet type, Hardy spaces, Bergman spaces, integral means, radial growth, sequences of zeros.

\section{Introduction and main results}

We denote by $\Delta$ the unit disc $\{z \in \mathbb{C}:|z|<1\}$. If $f$ is a function which is analytic in $\Delta$ and $0<r<1$, we set

$$
\begin{aligned}
M_{p}(r, f) & =\left(\frac{1}{2 \pi} \int_{-\pi}^{\pi}\left|f\left(r e^{i t}\right)\right|^{p} d t\right)^{1 / p}, \quad 0<p<\infty, \\
I_{p}(r, f) & =M_{p}^{p}(r, f), \quad 0<p<\infty, \\
M_{\infty}(r, f) & =\sup _{|z|=r}|f(z)| .
\end{aligned}
$$

For $0<p \leq \infty$, the Hardy space $H^{p}$ consists of all analytic functions $f$ in the disc for which $\|f\|_{H^{p}} \stackrel{\text { def }}{=} \sup _{0<r<1} M_{p}(r, f)<\infty$. We refer the reader to [10] and [13] for the theory of Hardy spaces.

(C) 2006 Australian Mathematical Society $1446-7887 / 06 \$ \mathrm{~A} 2.00+0.00$ 
If $0<p<\infty$ and $\alpha>-1$, we let $A_{\alpha}^{p}$ denote the (standard) weighted Bergman space, that is, the set of analytic functions $f$ in $\Delta$ such that

$$
\int_{\Delta}(1-|z|)^{\alpha}|f(z)|^{p} d A(z)<\infty .
$$

Here, $d A(z)=(1 / \pi) d x d y$ denotes the normalized Lebesgue area measure in $\Delta$. The standard unweighted Bergman space $A_{0}^{p}$ is simply denoted by $A^{p}$. We mention [11] and [17] as general references for the theory of Bergman spaces.

The space $\mathscr{D}_{\alpha}^{p}(p>0, \alpha>-1)$ consists of all functions $f$ which are analytic in $\Delta$ such that $f^{\prime} \in A_{\alpha}^{p}$. The space $\mathscr{D}_{0}^{2}$ is the classical Dirichlet space $\mathscr{D}$. For other values of $p$ and $\alpha$ the spaces $\mathscr{D}_{\alpha}^{p}$ have been extensively studied in a number papers such as $[27,28,30,33]$ for $p=2$ and $[4,8,34,36]$ for other values of $p$. If $p<\alpha+1$, it is well known that $\mathscr{D}_{\alpha}^{p}=A_{\alpha-p}^{p}$ with equivalence of norms (see [12, Theorem 6]). For $\alpha=p-2$, the space $\mathscr{D}_{\alpha}^{p}$ is the Besov space $B^{p}$ (compare to [3]).

The space $\mathscr{D}_{\alpha}^{p}$ is said to be a Dirichlet space if $p \geq \alpha+1$. In this paper we shall be primarily interested in the 'limit case' $p=\alpha+1$, that is, in the spaces $\mathscr{D}_{p-1}^{p}$, $0<p<\infty$, which are closely related to Hardy spaces. Indeed, a classical result of Littlewood and Paley [19] (see also [20]) asserts that

$$
H^{p} \subset \mathscr{D}_{p-1}^{p}, \quad 2 \leq p<\infty .
$$

On the other hand, we have

$$
\mathscr{D}_{p-1}^{p} \subset H^{p}, \quad 0<p \leq 2,
$$

(see [34, Lemma 1.4]). Notice that, in particular, we have $\mathscr{D}_{1}^{2}=H^{2}$. However, we remark that if $p \neq 2$ then

$$
H^{p} \neq \mathscr{D}_{p-1}^{p}
$$

This can be seen using the characterization of power series with Hadamard gaps which belong to the spaces $\mathscr{D}_{p-1}^{p}$.

PROPOSITION A. If $f$ is an analytic function in $\Delta$ which is given by a power series with Hadamard gaps, $f(z)=\sum_{k=1}^{\infty} a_{k} z^{n_{k}}(z \in \Delta)$ with $n_{k+1} \geq \lambda n_{k}$ for all $k(\lambda>1)$, then, for every $p \in(0, \infty), f \in \mathscr{D}_{p-1}^{p}$ if and only if $\sum_{k=1}^{\infty}\left|a_{k}\right|^{p}<\infty$.

Since for Hadamard gap series as above we have, for $0<p<\infty, f \in H^{p}$ if and only of $\sum_{k=1}^{\infty}\left|a_{k}\right|^{2}<\infty$, we immediately deduce that $\mathscr{D}_{p-1}^{p} \neq H^{p}$ if $p \neq 2$. We remark that Proposition A follows from [7, Proposition 2.1]. In Section 2 we shall see that Proposition A can also be deduced from the following theorem which gives a condition on the Taylor coefficients of a function $f$, analytic in $\Delta$, which implies that $f \in \mathscr{D}_{p-1}^{p}$. 
THEOREM 1.1. Let $f$ be an analytic function in $\Delta, f(z)=\sum_{n=0}^{\infty} a_{n} z^{n}(z \in \Delta)$.

(i) If $0<p<\infty$ and

$$
\sum_{n=0}^{\infty}\left(\sum_{k \in I(n)}\left|a_{k}\right|\right)^{p}<\infty,
$$

then $f \in \mathscr{D}_{p-1}^{p}$.

(ii) If $0<p \leq 2$ and

$$
\sum_{n=1}^{\infty}\left(\sum_{k \in I(n)}\left|a_{k}\right|^{2}\right)^{p / 2}<\infty
$$

then $f \in \mathscr{D}_{p-1}^{p}$.

Here and throughout the paper, for $n=0,1, \ldots, I(n)$ is the set of the integers $k$ such that $2^{n} \leq k<2^{n+1}$.

If $0<p \leq 2$, then (4) implies (5). Hence, for $p \in(0,2]$, (ii) is stronger than (i). We remark also that if $0<p \leq 2$, then the condition $\sum_{n=0}^{\infty}\left|a_{n}\right|^{p}<\infty$ implies (5). Consequently, (ii) improves [34, Lemma 1.5].

In Theorem 1.2 we give a condition on the Taylor coefficients of an analytic function $f$ which is necessary for its membership in $\mathscr{D}_{p-1}^{p}$ if $2 \leq p<\infty$.

THEOREM 1.2. Let $f$ be an analytic function in $\Delta, f(z)=\sum_{n=0}^{\infty} a_{n} z^{n}(z \in \Delta)$. If $2 \leq p<\infty$ and $f \in \mathscr{D}_{p-1}^{p}$, then

$$
\sum_{n=1}^{\infty}\left(\sum_{k \in I(n)}\left|a_{k}\right|^{2}\right)^{p / 2}<\infty .
$$

If $0<p<2$ then (3) can be seen in some other ways. Rudin proved in [29] that there exists a Blaschke product $B$ which does not belong to $\mathscr{D}_{0}^{1}$ (see also [24]). Vinogradov [34] extended this result showing that for every $p \in(0,2)$ there exist Blaschke products $B$ which do not belong to $\mathscr{D}_{p-1}^{p}$. This clearly gives that $\mathscr{D}_{p-1}^{p} \neq H^{p}$ if $0<p<2$, a fact which can be also deduced from the results of [9] and [14]. In contrast with what happens for $0<p<2$, it is not easy to give examples of functions $f \in \mathscr{D}_{p-1}^{p} \backslash H^{p}$ for a certain $p \in(2, \infty)$ that are not given by power series by Hadamard gaps. Since $H^{p} \subset \mathscr{D}_{p-1}^{p}$ if $p \geq 2$, any Blaschke product belongs to $\bigcap_{2 \leq p<\infty} \mathscr{D}_{p-1}^{p}$. Also, for a number of classes $\mathscr{F}$ of analytic functions in $\Delta$ we have $\mathscr{F} \cap \mathscr{D}_{p-1}^{p}=\mathscr{F} \cap H^{p}(0<p<\infty)$. For example, it is very easy to prove the following lemma.

LEMMA 1.3. (i) If $\alpha>0,0<p<\infty$, and $f(z)=1 /(1-z)^{\alpha},(z \in \Delta)$, then $f \in H^{p}$ if and only if $f \in \mathscr{D}_{p-1}^{p}$ if and only if $\alpha p<1$. 
(ii) If $\alpha, \beta>0, p \in(0, \infty)$, and

$$
f(z)=\frac{1}{(1-z)^{\alpha}\left(\log (2 /(1-z))^{\beta}\right.}, \quad(z \in \Delta),
$$

then $f \in H^{p}$ if and only if $f \in \mathscr{D}_{p-1}^{p}$ if and only if $\alpha p<1$ and $\beta>0$ or $\alpha p=1$ and $\beta p>1$.

A much deeper result is stated in [6, Theorem 1] which asserts that, if $\mathscr{U}$ denotes the class of all univalent (holomorphic and one-to-one) functions in $\Delta$, then $\mathscr{U} \cap H^{p}=$ $\mathscr{U} \cap \mathscr{D}_{p-1}^{p}$ for all $p>0$ (see also [25] for the case $p=1$ ).

In spite of these facts we shall prove that, for every $p \in(2, \infty)$, there are a lot of differences between the space $H^{p}$ and the space $\mathscr{D}_{p-1}^{p}$. In Section 3 , we shall be mainly concerned in obtaining sharp estimates on the growth of the integral means of $\mathscr{D}_{p-1}^{p}$-functions. If $0<p \leq 2$ and $f \in \mathscr{D}_{p-1}^{p}$, then $f \in H^{p}$ and hence, the integral means $M_{p}(r, f)$ are bounded. This is no longer true for $p>2$. Our main results in Section 3 are stated in the following two theorems.

THEOREM 1.4. If $2<p<\infty$ and $f \in \mathscr{D}_{p-1}^{p}$, then

(i)

$$
M_{p}(r, f)=\mathrm{O}\left(\left(\log \frac{1}{1-r}\right)\right), \quad \text { as } r \rightarrow 1 .
$$

$$
M_{2}(r, f)=\mathrm{O}\left(\left(\log \frac{1}{1-r}\right)^{1 / 2-1 / p}\right), \quad \text { as } r \rightarrow 1
$$

THEOREM 1.5. If $2<p<\infty$ and $0<\beta<1 / 2-1 / p$, then there exists a function $f \in \mathscr{D}_{p-1}^{p}$ such that

$$
\exp \left(\frac{1}{2 \pi} \int_{-\pi}^{\pi} \log \left|f\left(r e^{i t}\right)\right| d t\right) \neq \mathrm{o}\left(\left(\log \frac{1}{1-r}\right)^{\beta}\right), \quad \text { as } r \rightarrow 1^{-}
$$

Since

$$
\exp \left(\frac{1}{2 \pi} \int_{-\pi}^{\pi} \log \left|f\left(r e^{i t}\right)\right| d t\right) \leq M_{2}(r, f)
$$

Theorem 1.5 shows that part (ii) of Theorem 1.4 is sharp in a very strong sense. 
REMARK. Using Theorem 1.4 we can obtain an upper bound on the integral means $M_{q}(r, f), 2<q<p$, of a function $f \in \mathscr{D}_{p-1}^{p}$. Indeed, if $q \in(2, p)$, then $q=p \lambda+2(1-\lambda)$, where $\lambda=(q-2) /(p-2) \in(0,1)$. Consequently, using Theorem 1.4 and Hölder's inequality with exponents $1 / \lambda$ and $1 /(1-\lambda)$ we see that, if $f \in \mathscr{D}_{p-1}^{p}$ and $2<q<p$, then

$$
M_{q}(r, f)=\left(\left(\log \frac{1}{1-r}\right)^{\eta}\right), \quad \text { as } r \rightarrow 1,
$$

where $\eta=\eta(p, q)=p \lambda / q+(p-2)(1-\lambda) / p q$ and $\lambda=(q-2) /(p-2)$.

In Section 4 we study properties of the sequences of zeros of non trivial $\mathscr{D}_{p-1}^{p}$ functions. If $0<p \leq 2$ then $\mathscr{D}_{p-1}^{p} \subset H^{p}$ and hence, the sequence of zeros of a non-identically zero $\mathscr{D}_{p-1}^{p}$-function satisfies the Blaschke condition. This does not remain true for $p>2$. Our main results about the sequences of zeros of functions $f$ in the space $\mathscr{D}_{p-1}^{p}, 2<p<\infty$, are stated in Theorem 1.6 and Theorem 1.7

THEOREM 1.6. Suppose that $2<p<\infty$ and let $f$ be a function which belongs to the space $\mathscr{D}_{p-1}^{p}$ with $f(0) \neq 0$. Let $\left\{z_{k}\right\}_{k=1}^{\infty}$ be the sequence zeros of $f$ ordered so that $\left|z_{k}\right| \leq\left|z_{k+1}\right|$ for all $k$. Then

$$
\prod_{k=1}^{N} \frac{1}{\left|z_{k}\right|}=\mathrm{o}\left((\log N)^{1 / 2-1 / p}\right), \quad \text { as } N \rightarrow \infty .
$$

From now on, if $f$ is a non-identically zero analytic function of zeros and $\left\{z_{k}\right\}_{k=1}^{\infty}$ is the sequence zeros of $f$ ordered so that $\left|z_{k}\right| \leq\left|z_{k+1}\right|$ for all $k$, we shall say that $\left\{z_{k}\right\}_{k=1}^{\infty}$ is the sequence of ordered zeros of $f$. Theorem 1.7 asserts that Theorem 1.6 is best possible.

THEOREM 1.7. If $2<p<\infty$ and $0<\beta<1 / 2-1 / p$, then there exists a function $f \in \mathscr{D}_{p-1}^{p}$ with $f(0) \neq 0$ such that if $\left\{z_{k}\right\}_{k=1}^{\infty}$ is the sequence of ordered zeros of $f$, then

$$
\prod_{k=1}^{N} \frac{1}{\left|z_{k}\right|} \neq \mathrm{o}\left((\log N)^{\beta}\right), \quad \text { as } N \rightarrow \infty .
$$

As a consequence of Theorem 1.6 and Theorem 1.7, we obtain the following result.

COROLLARY 1.8. If $2 \leq p<q<\infty$ then there exists a sequence $\left\{z_{k}\right\} \subset \Delta$ that is the sequence of zeros of a $\mathscr{D}_{q-1}^{q}$-function but is not the sequence of zeros of any $\mathscr{D}_{p-1}^{p}$-function. 
Hence the situation in this setting is similar to that in the setting of Bergman spaces (see [18, Theorem 1]).

Next we shall get into the proofs of these and some other results. We shall be using the convention that $C_{p, \alpha, \ldots}$ denotes a positive constant which depends only upon the displayed parameters $p, \alpha, \ldots$ but is not necessarily the same at different occurrences.

\section{Taylor coefficients of $\mathscr{D}_{p-1}^{p}$ functions.}

We start by recalling the following useful result due to Mateljevic and Pavlovic [21] (see also [5, Lemma 3] and [22]) which will be basic in the proofs of Theorem 1.1 and Theorem 1.2.

LEMMA B. Let $\alpha>0$ and $p>0$. There exists a constant $K$ that depends only on $p$ and $\alpha$ such that, if $\left\{a_{n}\right\}_{n=1}^{\infty}$ is a sequence of non-negative numbers, $t_{n}=\sum_{k \in I(n)} a_{n}$ $(n \geq 0)$, and $f(x)=\sum_{n=1}^{\infty} a_{n} x^{n-1}(x \in(0,1))$, then

$$
K^{-1} \sum_{n=0}^{\infty} 2^{-n \alpha} t_{n}^{p} \leq \int_{0}^{1}(1-x)^{\alpha-1} f(x)^{p} d x \leq K \sum_{n=0}^{\infty} 2^{-n \alpha} t_{n}^{p} .
$$

ProOF OF TheOREM 1.1. Take $p \in(0, \infty)$ and let $f$ be analytic in $\Delta$,

$$
f(z)=\sum_{n=0}^{\infty} a_{n} z^{n}, \quad z \in \Delta .
$$

Suppose that (4) holds. Using Lemma B and (4) we see that

$$
\begin{aligned}
\int_{\Delta}\left|f^{\prime}(z)\right|^{p}\left(1-|z|^{2}\right)^{p-1} d A(z) & \leq C_{p} \int_{0}^{1}(1-r)^{p-1}\left(\sum_{n=1}^{\infty} n\left|a_{n}\right| r^{n-1}\right)^{p} d r \\
& \leq C_{p} \sum_{n=0}^{\infty} 2^{-n p}\left(\sum_{k \in I(n)} k\left|a_{k}\right|\right)^{p} \\
& \leq C_{p} \sum_{n=0}^{\infty} 2^{-n p} 2^{(n+1) p}\left(\sum_{k \in I(n)}\left|a_{k}\right|\right)^{p} \\
& \leq C_{p} \sum_{n=0}^{\infty}\left(\sum_{k \in I(n)}\left|a_{k}\right|\right)^{p}<\infty .
\end{aligned}
$$

Hence, $f \in \mathscr{D}_{p-1}^{p}$ and the proof of (i) is finished. 
Suppose now that $0<p \leq 2, f$ is as in (12) and satisfies (5). Using the fact that $M_{p}\left(r, f^{\prime}\right) \leq M_{2}\left(r, f^{\prime}\right)$ for all $r \in(0,1)$, making the change of variable $r^{2}=s$ and using Lemma B, we obtain

$$
\begin{aligned}
\int_{\Delta}\left|f^{\prime}(z)\right|^{p}\left(1-|z|^{2}\right)^{p-1} d A(z) & =2 \int_{0}^{1} r\left(1-r^{2}\right)^{p-1} M_{p}\left(r, f^{\prime}\right)^{p} d r \\
& \leq 2 \int_{0}^{1} r\left(1-r^{2}\right)^{p-1} M_{2}\left(r, f^{\prime}\right)^{p} d r \\
& =2 \int_{0}^{1} r\left(1-r^{2}\right)^{p-1}\left(\sum_{n=1}^{\infty} n^{2}\left|a_{n}\right|^{2} r^{2 n-2}\right)^{p / 2} d r \\
& \leq C \int_{0}^{1}(1-s)^{p-1}\left(\sum_{n=1}^{\infty} n^{2}\left|a_{n}\right|^{2} s^{n-1}\right)^{p / 2} d s \\
& \leq C_{p} \sum_{n=0}^{\infty} 2^{-n p}\left(\sum_{k \in I(n)} k^{2}\left|a_{k}\right|^{2}\right)^{p / 2} \\
& \leq C_{p} \sum_{n=0}^{\infty}\left(\sum_{k \in I(n)}\left|a_{k}\right|^{2}\right)^{p / 2}<\infty .
\end{aligned}
$$

Hence, $f \in \mathscr{D}_{p-1}^{p}$. This finishes the proof of (ii).

Next we see that Proposition A can be deduced from Theorem 1.1 as announced.

ProOF OF PROPOSITION A. Let $f$ be an analytic function in $\Delta$ given by a power series with Hadamard gaps

$$
f(z)=\sum_{j=1}^{\infty} a_{j} z^{n_{j}} \quad \text { with } \quad \frac{n_{j+1}}{n_{j}} \geq \lambda>1 \quad \text { for all } j,
$$

and suppose that $\sum_{j=1}^{\infty}\left|a_{j}\right|^{p}<\infty$. Using the gap condition, we see that there are at most $C_{\lambda}=\log _{\lambda} 2+1$ of the $n_{j}^{\prime} s$ in the set $I(n)$. Then there exists a constant $C_{\lambda, p}>0$ such that

$$
\sum_{n=0}^{\infty}\left(\sum_{j \in I(n)}\left|a_{j}\right|\right)^{p} \leq C_{\lambda, p} \sum_{j=1}^{\infty}\left|a_{j}\right|^{p}<\infty,
$$

and consequently, using Theorem 1.1, we deduce that $f \in \mathscr{D}_{p-1}^{p}$.

To prove the other implication suppose that $f$ is as in (13) and $f \in \mathscr{D}_{p-1}^{p}$ for a certain $p>0$. It is well known (see [38, Chapter V, Vol. I]) that there exist constants $A(\lambda, p)$ and $B(\lambda, p)$ such that

$$
A(\lambda, p) M_{2}^{p}\left(r, f^{\prime}\right) \leq M_{p}^{p}\left(r, f^{\prime}\right) \leq B(\lambda, p) M_{2}^{p}\left(r, f^{\prime}\right), \quad 0<r<1 .
$$


This and Lemma $\mathrm{B}$ give

$$
\begin{aligned}
\infty & >\int_{\Delta}\left|f^{\prime}(z)\right|^{p}\left(1-|z|^{2}\right)^{p-1} d A(z)=\int_{0}^{1} r\left(1-r^{2}\right)^{p-1} M_{p}^{p}\left(r, f^{\prime}\right) d r \\
& \geq A(\lambda, p) \int_{0}^{1} r\left(1-r^{2}\right)^{p-1} M_{2}^{p}\left(r, f^{\prime}\right) d r \\
& \geq A(\lambda, p) \int_{0}^{1} r\left(1-r^{2}\right)^{p-1}\left(\sum_{j=1}^{\infty} n_{j}{ }^{2}\left|a_{j}\right|^{2} r^{2 n_{j}-2}\right)^{p / 2} d r \\
& \geq A(\lambda, p) \int_{0}^{1} t(1-t)^{p-1}\left(\sum_{j=1}^{\infty} n_{j}{ }^{2}\left|a_{j}\right|^{2} t^{j-1}\right)^{p / 2} d t \\
& \geq C_{p} A(\lambda, p) \sum_{n=0}^{\infty} 2^{-n p}\left(\sum_{n_{j} \in I(n)} n_{j}{ }^{2}\left|a_{j}\right|^{2}\right)^{p / 2} \\
& \geq C_{p} A(\lambda, p) \sum_{n=0}^{\infty} 2^{-n p} 2^{n p}\left(\sum_{n_{j} \in I(n)}\left|a_{j}\right|\right)^{p} \geq C_{\lambda, p} A(\lambda, p) \sum_{j=0}^{\infty}\left|a_{j}\right|^{p} .
\end{aligned}
$$

The last inequality is obvious if $p \geq 1$ and, in the case $0<p<1$, follows again using the fact that there are at most $C_{\lambda}=\log _{\lambda} 2+1$ of the $n_{j}^{\prime} s$ in the set $I(n)$. Thus, we have $\sum_{j=0}^{\infty}\left|a_{j}\right|^{p}<\infty$. This finishes the proof.

PROOF OF THEOREM 1.2. Suppose that $2 \leq p<\infty$ and $f \in \mathscr{D}_{p-1}^{p}$,

$$
f(z)=\sum_{n=0}^{\infty} a_{n} z^{n}, \quad z \in \Delta .
$$

Using Lemma $\mathrm{B}$, bearing in mind that $k \asymp 2^{n}$ if $k \in I(n)$, making a change of variable, and using that since $p \geq 2, M_{2}\left(r, f^{\prime}\right) \leq M_{p}\left(r, f^{\prime}\right)$, we obtain

$$
\begin{aligned}
\sum_{n=1}^{\infty}\left(\sum_{k \in I(n)}\left|a_{k}\right|^{2}\right)^{p / 2} & \leq \sum_{n=1}^{\infty} 2^{-n p}\left(\sum_{k \in I(n)} k^{2}\left|a_{k}\right|^{2}\right)^{p / 2} \\
& \leq C_{p} \int_{0}^{1}(1-t)^{p-1}\left(\sum_{n=1}^{\infty} n^{2}\left|a_{n}\right|^{2} t^{n-1}\right)^{p / 2} d t \\
& \leq C_{p} \int_{0}^{1}\left(1-r^{2}\right)^{p-1}\left(\sum_{n=1}^{\infty} n^{2}\left|a_{n}\right|^{2} r^{2 n-2}\right)^{p / 2} d t \\
& \leq C_{p} \int_{0}^{1}(1-r)^{p-1} M_{p}\left(r, f^{\prime}\right)^{p}<\infty .
\end{aligned}
$$




\section{Growth properties of $\mathscr{D}_{p-1}^{p}$-functions}

In this section we are mainly interested in obtaining sharp estimates on the growth of functions $f$ in the spaces $\mathscr{D}_{p-1}^{p}(2<p<\infty)$.

3.1. Integral means estimates Let us start with estimates on the growth of the maximum modulus $M_{\infty}(r, f)$. We can prove the following result.

THEOREM 3.1. Let $f$ be an analytic function in $\Delta$. If $f \in \mathscr{D}_{p-1}^{p}, 0<p<\infty$, then

$$
M_{\infty}(r, f)=\mathrm{o}\left(\frac{1}{(1-r)^{1 / p}}\right), \quad \text { as } r \rightarrow 1^{-} \text {. }
$$

Proof. Let $f \in \mathscr{D}_{p-1}^{p}$ and $z \in \Delta$. Let $D(z)$ denote the open disc

$$
\left\{w \in \mathbb{C}:|z-w|<\frac{1-|z|}{2}\right\}
$$

Clearly, $D(z) \subset \Delta$. Since the function $z \rightarrow\left|f^{\prime}(z)\right|^{p}$ is subharmonic in $\Delta$, we have

$$
\left|f^{\prime}(z)\right|^{p} \leq \frac{C}{|D(z)|} \int_{D(z)}\left|f^{\prime}(\omega)\right|^{p} d A(\omega) \leq \frac{C}{\left(1-|z|^{2}\right)^{2}} \int_{D(z)}\left|f^{\prime}(\omega)\right|^{p} d A(\omega) .
$$

It is clear that $\left(1-|z|^{2}\right) \asymp\left(1-|\omega|^{2}\right), \omega \in D(z), z \in \Delta$. Using this and (15) we obtain

$$
\begin{aligned}
\left|f^{\prime}(z)\right|^{p} & \leq \frac{C_{p}}{\left(1-|z|^{2}\right)^{2}} \int_{D(z)}\left[\frac{1-|\omega|}{1-|z|}\right]^{p-1}\left|f^{\prime}(\omega)\right|^{p} d A(\omega) \\
& =\frac{C_{p}}{\left(1-|z|^{2}\right)^{p+1}} \int_{D(z)}(1-|\omega|)^{p-1}\left|f^{\prime}(\omega)\right|^{p} d A(\omega) .
\end{aligned}
$$

On the other hand, since $f \in \mathscr{D}_{p-1}^{p}$, it follows that

$$
\int_{D(2)}(1-|\omega|)^{p-1}\left|f^{\prime}(\omega)\right|^{p} d A(\omega)=o(1), \quad \text { as }|z| \rightarrow 1^{-}
$$

which, with (16), implies

$$
M_{\infty}\left(r, f^{\prime}\right)=o\left(\frac{1}{(1-r)^{1+1 / p}}\right), \quad \text { as } r \rightarrow 1^{-},
$$

and (14) follows by integration. 
REMARK. We observe that for any $p \in(0, \infty)$, the exponent $1 / p$ in $(14)$ is the best possible. Moreover, if we take

$$
f_{p, \beta}(z)=(1-z)^{-1 / p}\left(\log \frac{2}{1-z}\right)^{-\beta}, \quad z \in \Delta,
$$

with $\beta>\frac{1}{p}$ then, as we noticed in Lemma $1.3, f_{p . \beta} \in \mathscr{D}_{p-1}^{p}$ and it is easy to see that

$$
M_{\infty}(r, f) \approx(1-r)^{-1 / p}\left(\log \frac{1}{1-r}\right)^{-\beta}, \quad 0<r<1 .
$$

So condition (14) in Theorem 3.1 cannot be substituted by the condition

$$
M_{\infty}(r, f)=\mathrm{o}\left(\frac{1}{(1-r)^{1 / p}\left(\log (1 /(1-r))^{1 / p+\varepsilon}\right.}\right), \quad \text { as } r \rightarrow 1^{-},
$$

for any $\varepsilon>0$.

Now we turn to the proofs of Theorem 1.4 and Theorem 1.5.

ProOF OF THEOREM 1.4. Suppose that $2<p<\infty$ and $f \in \mathscr{D}_{p-1}^{p}$. Then

$$
\lim _{r \rightarrow 1^{-}} \int_{r}^{1}(1-s)^{p-1} M_{p}^{p}\left(s, f^{\prime}\right) d s=0 .
$$

Since $M_{p}\left(s, f^{\prime}\right)$ is an increasing function of $s$

$$
\int_{r}^{1}(1-s)^{p-1} M_{p}^{p}\left(s, f^{\prime}\right) d s \geq M_{p}^{p}\left(r, f^{\prime}\right) \int_{r}^{1}(1-s)^{p-1} d s \geq C_{p} M_{p}^{p}\left(r, f^{\prime}\right)(1-r)^{p},
$$

which, together with (18), yields

$$
M_{p}\left(r, f^{\prime}\right)=o\left((1-r)^{-1}\right), \quad \text { as } r \rightarrow 1^{-},
$$

which, using Minkowski's integral inequality, implies (7).

Using (19) and the fact that for any fixed $r$ with $0<r<1$ the integral means $M_{p}\left(r, f^{\prime}\right)$ increase with $p$, we deduce that

$$
I_{2}\left(r, f^{\prime}\right)=o\left((1-r)^{-2}\right), \quad \text { as } r \rightarrow 1^{-} .
$$

and then using the well-known inequality (see [26, pages 125-126])

$$
\frac{d^{2}}{d r^{2}}\left(I_{2}(r, f)\right) \leq 4 I_{2}\left(r, f^{\prime}\right), \quad 0<r<1,
$$


we obtain

$$
\frac{d^{2}}{d r^{2}}\left(I_{2}(r, f)\right)=\mathrm{o}\left((1-r)^{-2}\right) \quad \text { as } r \rightarrow 1^{-},
$$

which, integrating twice, gives

$$
M_{2}(r, f)=\mathrm{o}\left(\left(\log (1 /(1-r))^{1 / 2}\right), \quad \text { as } r \rightarrow 1 .\right.
$$

This is worse than (8). To obtain this we use Theorem 1.2.

Say that $f(z)=\sum_{n=1}^{\infty} a_{n} z^{n},(z \in \Delta)$. Suppose, without loss of generality that $a_{0}=0$. Using Hölder's inequality with the exponents $p / 2$ and $p /(p-2)$ and Theorem 1.2, we obtain

$$
\begin{aligned}
M_{2}(r, f)^{2} & =\sum_{n=1}^{\infty}\left|a_{n}\right|^{2} r^{2 n}=\sum_{n=0}^{\infty} \sum_{k \in I(n)}\left|a_{k}\right|^{2} r^{2 k} \leq \sum_{n=0}^{\infty} r^{2^{n+1}}\left(\sum_{k \in I(n)}\left|a_{k}\right|^{2}\right) \\
& \leq\left[\sum_{n=0}^{\infty}\left(\sum_{k \in I(n)}\left|a_{k}\right|^{2}\right)^{p / 2}\right]^{2 / p}\left[\sum_{n=0}^{\infty} r^{2^{n+1} p /(p-2)}\right]^{1-2 / p} \\
& \leq C_{f, p}\left(\log \frac{1}{1-r}\right)^{1-2 / p} .
\end{aligned}
$$

Since

$$
\exp \left(\frac{1}{2 \pi} \int_{-\pi}^{\pi} \log \left|f\left(r e^{i \theta}\right)\right| d \theta\right) \leq M_{2}(r, f), \quad 0<r<1,
$$

we trivially have the following result.

COROLlaRY 3.2. If $2<p<\infty$ and $f \in \mathscr{D}_{p-1}^{p}$, then

$$
\exp \left(\frac{1}{2 \pi} \int_{-\pi}^{\pi} \log \left|f\left(r e^{i \theta}\right)\right| d \theta\right)=\mathrm{O}\left(\left(\log \frac{1}{1-r}\right)^{1 / 2-1 / p}\right), \quad \text { as } r \rightarrow 1 .
$$

Theorem 1.5 shows that Corollary 3.2 and the estimate (8) are sharp in a very strong sense. The following lemma, whose proof is simple and is omitted, will be used in the proof of Theorem 1.5 .

LEMMA 3.3. Let $f(z)=\sum_{n=0}^{\infty} a_{n} z^{n}$ be an analytic function in $\Delta$. If $0<\beta \leq 1$ and $\sum_{k=0}^{N}\left|a_{k}\right|^{2} \approx(\log N)^{\beta}$, as $N \rightarrow \infty$, then $I_{2}(r, f) \approx\left(\log (1-r)^{-1}\right)^{\beta}$ as $r \rightarrow 1^{-}$.

We make use of the technique introduced by Ullrich in [32]. Let us start introducing some notation.

Let $\omega=[0,1]^{\mathbb{N}}$ and $\omega_{1}, \omega_{2}, \ldots$ be 'the coordinate functions' $\omega_{j}: \Omega \rightarrow[0,1]$. Let $d \omega$ denote the product measure $\Omega$ derived from the Lebesgue measure on $[0,1]$. Now 
$\omega_{1}, \omega_{2}, \ldots$ are the Steinhaus variables (independent, identically distributed random variables uniformly distributed on $[0,1])$. Note that $\left\{e^{2 \pi i \omega_{j}}\right\}_{j=1}^{\infty}$ is an orthonormal set in $L^{2}(\Omega)$, hence, if $\sum_{j=1}^{\infty}\left|a_{j}\right|^{2}<\infty$, then $\sum_{j=1}^{\infty} a_{j} e^{2 \pi i \omega_{j}}$ is a well defined element of $L^{2}(\Omega)$ with $L^{2}$-norm $\left(\sum_{j=1}^{\infty}\left|a_{j}\right|^{2}\right)^{1 / 2}$. The following theorem is [32, Theorem 1].

THEOREM C. There exists $C>0$ such that for any sequence of complex numbers $\left\{a_{j}\right\}_{j=1}^{\infty}$ with $\sum_{j=1}^{\infty}\left|a_{j}\right|^{2}<\infty$, we have

$$
\exp \left[\int_{\Omega} \log \left|\sum_{j=1}^{\infty} a_{j} e^{2 \pi i \omega_{j}}\right| d \omega\right] \geq C\left(\sum_{j=1}^{\infty}\left|a_{j}\right|^{2}\right)^{1 / 2} .
$$

ProOF OF THEOREM 1.5. Suppose that $2<p<\infty$ and $0<\beta<1 / 2-1 / p$. Set $\varepsilon=1 / 2-1 / p-\beta$, hence, $\varepsilon>0$. We define the sequence $\left\{b_{j}\right\}_{j=1}^{\infty}$ as $b_{j}=j^{-1 / p-\varepsilon}$, $j=1,2, \ldots$ Now, for every $\omega \in \Omega$ we define

$$
f_{\omega}(z)=\sum_{j=1}^{\infty} b_{j} e^{2 \pi i \omega_{j}} z^{2^{j}}=\sum_{k=1}^{\infty} a_{k, \omega} z^{k}, \quad z \in \Delta .
$$

Since $\sum_{j=1}^{\infty}\left|b_{j}\right|^{p}<\infty$, using Proposition A we deduce that $f_{\omega} \in \mathscr{D}_{p-1}^{p}$ for every $\omega \in \Omega$.

We will see that for a.e. $\omega \in \Omega$

$$
\exp \left(\frac{1}{2 \pi} \int_{-\pi}^{\pi} \log \left|f_{\omega}\left(r e^{i t}\right)\right| d t\right) \neq \mathrm{o}\left((\log (1 /(1-r)))^{\beta}\right), \quad \text { as } r \rightarrow 1^{-} .
$$

This will finish the proof.

Suppose that (21) is false. Then there exists a measurable set $E \subset \Omega$ with positive measure and such that for all $\omega \in E$

$$
\exp \left(\frac{1}{2 \pi} \int_{-\pi}^{\pi} \log \left|f_{\omega}\left(r e^{i t}\right)\right| d t\right)=\mathrm{o}\left((\log (1 /(1-r)))^{\beta}\right), \quad \text { as } r \rightarrow 1^{-} .
$$

This is equivalent to saying that

$$
\lim _{r \rightarrow 1^{-}} \frac{1}{2 \pi} \int_{-\pi}^{\pi} \log \left[\frac{\left|f_{\omega}\left(r e^{i t}\right)\right|}{(\log (1 /(1-r)))^{\beta}}\right] d t=-\infty, \quad \omega \in E .
$$

On the other hand,

$$
\begin{aligned}
\left(\sum_{j=1}^{N}\left|b_{j}\right|^{2}\right)^{1 / 2} & =\left(\sum_{j=1}^{N} \frac{1}{j^{2 / p+2 \varepsilon}}\right)^{1 / 2} \\
& \sim\left(\int_{1}^{N} \frac{1}{x^{2 / p+2 \varepsilon}} d x\right)^{1 / 2} \sim N^{1 / 2-1 / p-\varepsilon}, \quad \text { as } N \rightarrow \infty .
\end{aligned}
$$


Thus, there exist $C>0$ and $N_{0}>0$ such that

$$
\left(\sum_{k=1}^{N}\left|a_{k, \omega}\right|^{2}\right)^{1 / 2} \leq C(\log N)^{1 / 2-1 / p-\varepsilon}, \quad N \geq N_{0} .
$$

Using (24) and Lemma 3.3, we deduce that

$$
M_{2}\left(r, f_{\omega}\right)=I_{2}\left(r, f_{\omega}\right)^{1 / 2} \leq C\left[\log \frac{1}{1-r}\right]^{1 / 2-1 / p-\varepsilon}, \quad 0<r<1, \quad \omega \in \Omega,
$$

which implies that for $0<r<1$ and $\omega \in \Omega$,

$$
\exp \left(\frac{1}{2 \pi} \int_{-\pi}^{\pi} \log \left|f_{\omega}\left(r e^{i t}\right)\right| d t\right) \leq C\left[\log \frac{1}{1-r}\right]^{1 / 2-1 / p-\varepsilon} .
$$

From this we deduce as in (23), that there exists $C>0$ such that

$$
\int_{-\pi}^{\pi} \log \left[\frac{\left|f_{\omega}\left(r e^{i t}\right)\right|}{(\log (1 /(1-r)))^{\beta}}\right] d t \leq C, \quad 0<r<1, \quad \omega \in \Omega .
$$

Bearing in mind that $E$ has positive measure, (26) and (23) imply

$$
\lim _{r \rightarrow 1^{-}} \int_{\Omega}\left[\int_{-\pi}^{\pi} \log \frac{\left|f_{\omega}\left(r e^{i t}\right)\right|}{(\log (1 /(1-r)))^{\beta}} d t\right] d \omega=-\infty
$$

For $N=1,2, \ldots$, let $\Omega_{N}=[0,1]^{N}$ and $m_{N}$ be the Lebesgue measure on $\Omega_{N}$. Observe now that, for any $N$, we have

$$
\begin{aligned}
\int_{\Omega_{N}} \log \left|f_{\omega}\left(r e^{i t}\right)\right| d m_{N}(\omega) \\
\quad=\int_{0}^{1} \cdots \int_{0}^{1} \log \left|\sum_{j=1}^{N} b_{j} r^{2 j} e^{i\left[2 \pi \omega_{j}+2^{j} t\right]}+\sum_{j=N+1}^{\infty} b_{j} r^{2^{j}} e^{i\left[2 \pi \omega_{j}+2^{j} t\right]}\right| d \omega_{1} d \omega_{2} \cdots d \omega_{N} \\
\quad=\int_{0}^{1} \cdots \int_{0}^{1} \log \left|\sum_{j=1}^{N} b_{j} r^{2 j} e^{2 \pi i \omega_{j}}+\sum_{j=N+1}^{\infty} b_{j} r^{2^{j}} e^{i\left[2 \pi \omega_{j}+2^{j} t\right]}\right| d \omega_{1} d \omega_{2} \cdots d \omega_{N} \text {, a.s. }
\end{aligned}
$$

Letting $N$ tend to $\infty$, we deduce that $\int_{\Omega} \log \left|f_{\omega}\left(r e^{i t}\right)\right| d \omega$ is independent of $t$. Then using (27) and Fubini's Theorem we obtain

$$
\lim _{r \rightarrow 1^{-}} \int_{\Omega} \log \frac{\left|f_{\omega}(r)\right|}{(\log (1 /(1-r)))^{\beta}} d \omega=-\infty .
$$


However, if we set $r_{N}=1-1 / 2^{N}, N=1,2, \ldots$, by Theorem $\mathrm{C}$ and the inequality

$$
e^{-1} \leq r_{N}^{2^{N}} \leq r_{N}^{2^{j}}, \quad 1 \leq j \leq N,
$$

we deduce that

$$
\begin{aligned}
\exp & {\left[\int_{\Omega} \log \left|f_{\omega}\left(r_{N}\right)\right| d \omega\right] } \\
& =\exp \left[\int_{\Omega} \log \mid \sum_{j=1}^{\infty} b_{j} e^{\left.2 \pi i \omega_{j} r_{N}^{2 j} \mid\right]}\right. \\
& \geq C\left(\sum_{j=1}^{\infty}\left|b_{j}\right|^{2}\left(r_{N}^{2^{j}}\right)^{2}\right)^{1 / 2} \geq C\left(\sum_{j=1}^{N}\left|b_{j}\right|^{2}\right)^{1 / 2}=C\left(\sum_{j=1}^{N} \frac{1}{j^{2 / p+2 \varepsilon}}\right)^{1 / 2} \\
& \geq C \frac{1}{N^{1 / p+\varepsilon-1 / 2}} \geq C\left(\log \frac{1}{1-r_{N}}\right)^{1 / 2-1 / p-\varepsilon}=C\left(\log \frac{1}{1-r_{N}}\right)^{\beta},
\end{aligned}
$$

which implies

$$
\int_{\Omega} \log \frac{\left|f_{\omega}\left(r_{N}\right)\right|}{\left(\log \left(1-r_{N}\right)^{-1}\right)^{\beta}} d \omega \geq \log C, \text { for all } N,
$$

which contradicts (28). Consequently, (21) is true and the proof is finished.

3.2. Radial growth of $\mathscr{D}_{p-1}^{p}$-functions In this section we obtain some estimates on the radial growth of $\mathscr{D}_{p-1}^{p}$-functions. If $0<p \leq 2$ and $f \in \mathscr{D}_{p-1}^{p}$, then $f \in H^{p}$ and so $f$ has nontangential limit a.e. $\mathbb{T}$. Therefore, we have: If $0<p \leq 2$ and $f \in \mathscr{D}_{p-1}^{p}$, then $\left|f\left(r e^{i \theta}\right)\right|=\mathrm{O}(1)$, as $r \rightarrow 1^{-}$for a.e. $e^{i t} \in \partial \Delta$.

Zygmund proved in [37] that if $f$ is an analytic function in $\Delta$, then

$$
\int_{0}^{r}\left|f^{\prime}\left(\rho e^{i t}\right)\right| d \rho=\mathrm{o}\left[\left(\log \frac{1}{1-r}\right)^{1 / 2}\right], \quad \text { as } r \rightarrow 1^{-} .
$$

for almost every point $e^{i t}$ in the Fatou set of $f, F_{f}$, which consists of those $e^{i t} \in \mathbb{T}$ such that $f$ has finite nontangential limit at $e^{i t}$. Obviously, (29) implies

$$
\left|f\left(r e^{i t}\right)\right|=\mathrm{o}\left[\left(\log \frac{1}{1-r}\right)^{1 / 2}\right], \quad \text { as } r \rightarrow 1^{-},
$$

If $2<p<\infty$, there are functions $f \in \mathscr{D}_{p-1}^{p}$ such that $F_{f}$ has Lebesgue measure equal to zero. Indeed, an analytic function $f$ given by a power series with Hadamard gaps whose sequence of Taylor coefficients $\left\{a_{k}\right\}$ belongs to $l^{p} \backslash l^{2}$, is a $\mathscr{D}_{p-1}^{p}$-function by Proposition A and $F_{f}$ has null Lebesgue measure (see [38, Chapter V]). In spite of this, we can prove the following result for $\mathscr{P}_{p-1}^{p}$-functions. 
THEOREM 3.4. If $2<p<\infty$ and $f \in \mathscr{D}_{p-1}^{p}$, then

$$
\left|f\left(r e^{i t}\right)\right|=o\left[\left(\log \frac{1}{1-r}\right)^{1-1 / p}\right], \quad \text { as } r \rightarrow 1^{-} \text {for a. e. } e^{i t} \in \partial \Delta .
$$

This is better that the a.e. estimate which can be deduced from (17).

PROOF OF THEOREM 3.4. Let $p$ and $f$ be as in the statement of the theorem. Then

$$
\int_{-\pi}^{\pi}\left(\int_{0}^{1}(1-r)^{p-1}\left|f^{\prime}\left(r e^{i t}\right)\right|^{p} d t\right) d r<\infty
$$

and it follows that the set $A$ of points $e^{i t} \in \partial \Delta$ for which

$$
\int_{0}^{1}(1-r)^{p-1}\left|f^{\prime}\left(r e^{i t}\right)\right|^{p} d t<\infty
$$

has Lebesgue measure equal to $2 \pi$.

Take and fix $e^{i t} \in A$. Take also $\varepsilon>0$. Then there exists $r_{\varepsilon} \in(0,1)$ such that

$$
\int_{r_{\varepsilon}}^{1}(1-s)^{p-1}\left|f^{\prime}\left(s e^{i t}\right)\right|^{p} d s<\varepsilon .
$$

Using (32) and Hölder's inequality with exponents $p$ and $p /(p-1)$, we obtain for $r_{\varepsilon}<r<1$,

$$
\begin{aligned}
\int_{0}^{r}\left|f^{\prime}\left(s e^{i t}\right)\right| d s & =\int_{0}^{r_{\varepsilon}}\left|f^{\prime}\left(s e^{i t}\right)\right| d s+\int_{r_{\varepsilon}}^{r}\left|f^{\prime}\left(s e^{i t}\right)\right| d s \\
& \leq C_{f . \varepsilon}+\int_{r_{\varepsilon}}^{r} \frac{(1-s)^{1-1 / p}}{(1-s)^{1-1 / p}}\left|f^{\prime}\left(s e^{i t}\right)\right| d s \\
& \leq C_{f, \varepsilon}+\left[\int_{r_{\varepsilon}}^{r}(1-s)^{p-1}\left|f^{\prime}\left(s e^{i t}\right)\right|^{p} d s\right]^{1 / p}\left[\int_{r_{\varepsilon}}^{r} \frac{d s}{(1-s)}\right]^{1-1 / p} \\
& \leq C_{f, \varepsilon}+\varepsilon\left(\log \frac{1}{1-r}\right)^{1-1 / p}
\end{aligned}
$$

Consequently, we have proved that

$$
\limsup _{r \rightarrow 1}\left(\log \frac{1}{1-r}\right)^{1 / p-1} \int_{0}^{r}\left|f^{\prime}\left(s e^{i t}\right)\right| d s \leq \varepsilon .
$$

Since $\varepsilon>0$ and $e^{i t} \in A$ are arbitrary, we have

$$
\int_{0}^{r}\left|f^{\prime}\left(s e^{i t}\right)\right| d s=0\left[\left(\log \frac{1}{1-r}\right)^{1-1 / p}\right], \quad \text { as } r \rightarrow 1^{-},
$$

for all $e^{i t} \in A$. This implies that (31) holds for all $e^{i t} \in A$, which has Lebesgue measure equal to $2 \pi$. This finishes the proof. 
We do not know whether or not the exponent $1-1 / p$ in Theorem 3.4 is sharp but we know that it cannot be substitutes by any exponent smaller than $1 / 2-1 / p$. Indeed, we can prove the following result.

THEOREM 3.5. If $2<p<\infty$, then there exists a function $f \in \mathscr{D}_{p-1}^{p}$ such that

$$
\lim _{r \rightarrow 1^{-}} \frac{\left|f\left(r e^{i t}\right)\right|}{\left(\log \frac{1}{1-r}\right)^{1 / 2-1 / p}\left(\log \log \frac{1}{1-r}\right)^{-1}}=\infty, \quad \text { for a.e. } e^{i t} \in \partial \Delta .
$$

Proof. Take $p>2$. Define

$$
a_{k}=\frac{1}{k^{1 / p} \log 2 k}, \quad k=1,2, \ldots, \quad \text { and } \quad f(z)=\sum_{k=1}^{\infty} a_{k} z^{2^{k}}, \quad z \in \Delta .
$$

Since $\sum_{k=1}^{\infty}\left|a_{k}\right|^{p}<\infty$, by Proposition A, we have that $f \in \mathscr{D}_{p-1}^{p}$.

On the other hand,

$$
\begin{aligned}
\left(\sum_{k=1}^{N}\left|a_{k}\right|^{2}\right)^{1 / 2} & =\left(\sum_{k=1}^{N} \frac{1}{k^{2 / p} \log ^{2} 2 k}\right)^{1 / 2} \\
& \sim\left(\int_{1}^{N} \frac{1}{x^{2 / p} \log ^{2} 2 x} d x\right)^{1 / 2} \sim \frac{N^{1 / 2-1 / p}}{\log N}, \quad \text { as } N \rightarrow \infty,
\end{aligned}
$$

and then it is easy to see that

$$
M_{2}(r, f)=I_{2}(r, f)^{1 / 2} \sim \frac{\left(\log \frac{1}{1-r}\right)^{1 / 2-1 / p}}{\log \log \frac{1}{1-r}}, \quad \text { as } r \rightarrow 1^{-} .
$$

Now, by the law of the iterated logarithm for lacunary series (see [35]) we have that

$$
\lim _{r \rightarrow 1^{+}} \frac{\left|f\left(r e^{i t}\right)\right|}{\left[I_{2}(r, f) \log \log \log I_{2}(r, f)\right]^{1 / 2}}=1, \quad \text { for a.e. } e^{i t} \in \partial \Delta .
$$

Now we observe that (36) and (35) imply (34). This finishes the proof.

\section{Zeros of $\mathscr{D}_{p-1}^{p}$ functions}

4.1. Products of the zeros of $\mathscr{D}_{p-1}^{p}$ functions We start by recalling the the following result due to Horowitz, (see [18, page 65]). 
LEMMA D. Let $f$ be an analytic function in $\Delta$ with $f(0) \neq 0$ and let $\left\{z_{k}\right\}$ be the sequence of ordered zeros of $f$. If $0<p<\infty, 0 \leq r<1$, and $N$ is a positive integer, then

$$
|f(0)|^{p} \prod_{k=1}^{N} \frac{r^{p}}{\left|z_{k}\right|^{p}} \leq M_{p}(r, f)^{p} .
$$

This lemma and the estimates for the integral means of $\mathscr{D}_{p-1}^{p}$-functions obtained in Section 3.1 are the basic ingredients in the proofs of Theorem 1.6 and Theorem 1.7. This method was used by Horowitz in [18] for the Bergman spaces and later by the first author of this paper, Nowak, and Waniurski in [15] for the Bloch space $\mathscr{B}$ and some other related spaces.

PROOF OF THEOREM 1.6. Let $p, f$, and $\left\{z_{k}\right\}_{k=1}^{\infty}$ be as in the statement of Theorem 1.6. Using Theorem 1.4, we see that $f$ satisfies (8) and using Lemma D with $p=2$, we deduce that

$$
\prod_{k=1}^{N} \frac{r}{\left|z_{k}\right|} \leq C M_{2}(r, f) \leq C\left(\log \frac{1}{1-r}\right)^{1 / 2-1 / p}, \quad \text { if } r \text { is close enough to } 1 .
$$

Now, taking $r=1-1 / N$ with $N$ big enough in (38) and bearing in mind that $(1-1 / N)^{N}>1 / 2 e$, we deduce that

$$
\prod_{k=1}^{N} \frac{1}{\left|z_{k}\right|} \leq C(\log N)^{1 / 2-1 / p}
$$

This finishes the proof.

Our next objective is to prove Theorem 1.7 which asserts that Theorem 1.6 is sharp. We start recalling some notation and facts from Nevanlinna theory (see $[16,23]$ or [31]) which will be needed in our proof.

Let $f$ be a non-constant analytic function in $\Delta$. For any $a \in \mathbb{C}$ and $0<r<1$, we denote by $n(r, a, f)$ the number of zeros $f-a$ in the disc $\{|z| \leq r\}$, where each zero is counted according to its multiplicity. We define also

(40) $N(r, a, f) \stackrel{\text { def }}{=} \int_{0}^{r} \frac{n(t, a, f)-n(0, a, f)}{t} d t+n(0, a, f) \log r, \quad 0<r<1$.

For simplicity, we shall write $n(r, f)=n(r, 0, f), N(r, f)=N(r, 0, f)$. The Nevanlinna characteristic function $T(r, f)$ is defined by

$$
T(r, f)=\frac{1}{2 \pi} \int_{-\pi}^{\pi} \log ^{+}\left|f\left(r e^{i \theta}\right)\right| d \theta, \quad 0<r<1 .
$$


The proximity function $m(r, a, f)$ is given by

$$
m(r, a, f) \stackrel{\text { def }}{=} \frac{1}{2 \pi} \int_{-\pi}^{\pi} \log ^{+} \frac{1}{\left|f\left(r e^{i t}\right)-a\right|} d t, \quad 0<r<1 .
$$

Now we can state the First Fundamental Theorem of Nevanlinna.

THEOREM E. Let $f$ be a non-constant analytic function in $\Delta$. Then

$$
m(r, a, f)+N(r, a, f)=T(r, f)+O(1), \quad \text { as } r \rightarrow 1^{-} .
$$

for every $a \in \mathbb{C}$.

Now we can prove the following result.

Proposition 4.1. If $2<p<\infty$ and $f$ is a non-constant $\mathscr{D}_{p-1}^{p}$-function, then

$$
n(r, a, f)=\mathrm{O}\left(\frac{1}{1-r} \log \log \frac{1}{1-r}\right), \quad \text { as } r \rightarrow 1^{-}, \text {for all } a \in \mathbb{C} .
$$

Proof. Using the arithmetic-geometric mean inequality we obtain

$$
\begin{aligned}
T(r, f) & \leq \frac{1}{4 \pi} \int_{-\pi}^{\pi} \log \left(\left|f\left(r e^{i t}\right)\right|^{2}+1\right) d t \\
& \leq \frac{1}{2} \log \left(\frac{1}{2 \pi} \int_{-\pi}^{\pi}\left(\left|f\left(r e^{i t}\right)\right|^{2}+1\right) d t\right) \leq \frac{1}{2} \log \left(l_{2}(r, f)+1\right),
\end{aligned}
$$

which, with part (ii) of Theorem 1.4, gives

$$
T(r, f)=\mathrm{O}\left(\log \log \frac{1}{1-r}\right), \quad \text { as } r \rightarrow 1^{-} .
$$

Using Theorem E, we deduce that

$$
N(r, a, f)=\mathrm{O}\left(\log \log \frac{1}{1-r}\right), \quad \text { as } r \rightarrow 1^{-}, \text {for all } a \in \mathbb{C} .
$$

Now, it is well known (see [2, page 22]) that this implies (41).

Now, we can proceed with the proof of Theorem 1.7.

ProOF OF THEOREM 1.7. Take $p$ and $\beta$ with $2<p<\infty$ and $0<\beta<1 / 2-1 / p$. Take $f \in \mathscr{T}_{p-1}^{p}$ with $f(0) \neq 0$ and

$$
\exp \left(\frac{1}{2 \pi} \int_{-\pi}^{\pi} \log \left|f\left(r e^{i t}\right)\right| d t\right) \neq \mathrm{o}\left(\left(\log \frac{1}{1-r}\right)^{\beta}\right), \quad \text { as } r \rightarrow 1^{-},
$$


such a function exists by Theorem 1.5. Using (44) we see that there exist a sequence $\left\{r_{j}\right\}_{j=1}^{\infty} \subset(0,1)$ with $r_{j} \uparrow 1$ and a positive constant $C$ (independent of $j$ ), such that

$$
\exp \left(\frac{1}{2 \pi} \int_{-\pi}^{\pi} \log \left|f\left(r_{j} e^{i t}\right)\right| d t\right) \geq C\left(\log \frac{1}{1-r_{j}}\right)^{\beta}, \quad j=1,2 \ldots
$$

We shall write $n(r)$ instead of $n(r, f)$ for simplicity. Using Jensen's formula (see [1, page 206]) and (45) we deduce that

$$
|f(0)| \prod_{k=1}^{n\left(r_{j}\right)} \frac{r_{j}}{\left|z_{k}\right|} \geq C\left(\log \frac{1}{1-r_{j}}\right)^{\beta}, \quad j=1,2 \ldots,
$$

which implies that

$$
n\left(r_{j}\right) \rightarrow \infty, \quad \text { as } j \rightarrow \infty .
$$

On the other hand, Proposition 4.1 implies that there exists $C>0$ such that

$$
n(r) \leq C \frac{1}{1-r} \log \log \frac{1}{1-r}, \quad \text { if } r \text { is sufficiently close to } 1 .
$$

This implies that

$$
\log n(r) \leq C \log \frac{1}{1-r}, \quad \text { if } r \text { is sufficiently close to } 1,
$$

which, together with (46), shows that there exists $j_{0} \in \mathbb{N}$ such that for every $j \geq j_{0}$

$$
|f(0)| \prod_{k=1}^{n\left(r_{j}\right)} \frac{r_{j}}{\left|z_{k}\right|} \geq C\left[\log n\left(r_{j}\right)\right]^{\beta} .
$$

This finishes the proof.

4.2. A substitute of Blaschke condition If $2<p<\infty$ the sequence $\left\{z_{k}\right\}$ of ordered zeros of a non trivial $\mathscr{T}_{p-1}^{p}$ function need not satisfy the Blaschke condition. Indeed, the Blaschke condition is equivalent to saying that $\prod_{n=1}^{N}\left(1 /\left|z_{n}\right|\right)=\mathrm{O}(1)$ and we have seen that this is not always true. Using Theorem 1.6 and arguing exactly as in the proof of [15, Theorem 5] we can prove the following result.

THEOREM 4.2. Let $2<p<\infty$ and $f \in \mathscr{D}_{p-1}^{p}$ with $f \not \equiv 0$. Let $\left\{z_{k}\right\}_{k=1}^{\infty}$ be the sequence of zeros of $f$. Then

$$
\sum_{\left|z_{k}\right|>1-1 / e}\left(1-\left|z_{k}\right|\right)\left(\log \log \frac{1}{1-\left|z_{k}\right|}\right)^{-\alpha}<\infty
$$

for all $\alpha>1$. 
Next, we shall prove that the condition $\alpha>1$ is needed in Theorem 4.2.

THEOREM 4.3. Let $2<p<\infty$. Then there exists a function $f \in \mathscr{D}_{p-1}^{p}$ with $f \not \equiv 0$, whose sequence of zeros $\left\{z_{k}\right\}_{k=1}^{\infty}$ satisfies

$$
\sum_{\left|z_{k}\right|>i-1 / e}\left(1-\left|z_{k}\right|\right)\left(\log \log \frac{1}{1-\left|z_{k}\right|}\right)^{-1}=\infty .
$$

PROOF. Set $g(z)=\sum_{k=1}^{\infty} k^{-(p+2) / 4 p} z^{2^{k}}, z \in \Delta$. Since $g$ is given by a power series with Hadamard gaps and $\sum_{k=1}^{\infty} k^{-(p+2) / 4}<\infty$, it follows that $g \in \mathscr{D}_{p-1}^{p}$.

We shall follow the argument of the proof of [15, Theorem 6]. Set

$$
r_{n}=1-2^{-n}, \quad n=1,2,3, \ldots
$$

It is easy to see that, for all sufficiently large $n, I_{2}\left(r_{n}, g\right) \geq C n^{1 / 2-1 / p}$, which, since $\log \left(1 /\left(1-r_{n}\right)\right)=n \log 2$, implies that

$$
I_{2}\left(r_{n}, g\right) \geq C\left(\log \frac{1}{1-r_{n}}\right)^{1 / 2-1 / p} \quad \text { if } n \text { is sufficiently large. }
$$

Now, since $\log \left(1 /\left(1-r_{n}\right)\right) \sim \log \left(1 /\left(1-r_{n+1}\right)\right)$, as $n \rightarrow \infty$, and since $I_{2}(r, g)$ and $(\log (1 /(1-r)))^{1 / 2-1 / p}$ are increasing functions of $r$, we deduce

$$
I_{2}(r, g) \geq C\left(\log \frac{1}{1-r}\right)^{1 / 2-1 / p},
$$

if $r$ is sufficiently close to 1 .

Using this and arguing as in [15, page 126] we deduce that there exist a complex number $a$ with $g(0) \neq a$, a positive constant $\beta$, and a number $r_{0} \in(0,1)$ such that

$$
N(r, a, g) \geq \beta \log \log \frac{1}{1-r} \quad r \in\left(r_{0}, 1\right) .
$$

Take such an $a \in \mathbb{C}$ and set $f(z)=g(z)-a, z \in \Delta$. Then $f \in \mathscr{D}_{p-1}^{p}$ and $f(0) \neq 0$. Also (53) can be written as

$$
N(r, f) \geq \beta \log \log \frac{1}{1-r}, \quad r \in\left(r_{0}, 1\right) .
$$

Let $\left\{z_{n}\right\}$ be the sequence of zeros of $f$. Using Proposition 4.1 and arguing as in [15, page 127], we obtain (49). 


\section{Acknowledgements}

We wish to thank the referee for his/her helpful remarks.

The authors have been supported in part by grants from 'El Ministerio de Educación y Ciencia', Spain (BFM2001-1736, MTM2004-00078 and MTM2004-21420-E) and by a grant from 'La Junta de Andalucía' (FQM-210).

\section{References}

[1] L. V. Ahlfors, Complex analysis, 2nd edition (Dover, McGraw-Hill, New York, 1966).

[2] J. M. Anderson, J. Clunie and Ch. Pommerenke, 'On Bloch functions and normal functions', J. Reine Angew. Math. 270 (1974), 12-37.

[3] J. Arazy, S. D. Fisher and J. Peetre, 'Möbius invariant function spaces', J. Reine Angew. Math. 363 (1985), 110-145.

[4] N. Arcozzi, R. Rochberg and E. Sawyer, 'Carleson measures for analytic Besov spaces', Rev. Mat. Iberoamericana 18 (2002), 443-510.

[5] R. Aulaskari, J. Xiao and R. Zhao, 'On subspaces and subsets of BMOA and UBC', Analysis 15 (1995), 101-121.

[6] A. Baernstein II, D. Girela and J. A. Peláez, 'Univalent functions, hardy spaces and spaces of Dirichlet type', Illinois J. Math. 48 (2004), 837-859.

[7] S. M. Buckley, P. Koskela and D. Vukotić, 'Fractional integration, differentiation, and weighted Bergman spaces', Math. Proc. Cambridge Philos. Soc. 126 (1999), 369-385.

[8] B. R. Choe, H. Koo and W. Smith, 'Composition operators acting on holomorphic Sobolev spaces', Trans. Amer. Math. Soc. 355 (2003), 2829-2855.

[9] E. S. Doubtsov, 'Corrected outer functions', Proc. Amer. Math. Soc. 126 (1998), 515-522.

[10] P. L. Duren, Theory of $H^{p}$ spaces, 2nd edition (Dover, Mineola, New York, 2000).

[11] P. L. Duren and A. P. Schuster, Bergman spaces, Math. Surveys and Monographs 100 (American Mathematical Society, Providence, RI, 2004).

[12] T. M. Flett, 'The dual of an inequality of Hardy and Littlewood and some related inequalities', J. Math. Anal. Appl. 38 (1972), 746-765.

[13] J. B. Garnett, Bounded analytic functions (Academic Press, New York, 1981).

[14] D. Girela, 'Growth of the derivative of bounded analytic functions', Complex Var. Theory Appl. 20 (1992), 221-227.

[15] D. Girela, M. Nowak and P. Waniurski, 'On the zeros of Bloch functions', Math. Proc. Camb. Philos. Soc. 129 (2001), 117-128.

[16] W. K. Hayman, Meromorphic functions, Oxford Mathematical Monographs (Clarendon Press, Oxford, 1964).

[17] H. Hedenmalm, B. Korenblum and K. Zhu, Theory of Bergman Spaces, Graduate Texts in Mathematics 199 (Springer, New York, 2000).

[18] C. Horowitz, 'Zeros of functions in Bergman spaces', Duke Math. J. 41 (1974), 693-710.

[19] J. E. Littlewood and R. E. A. C. Paley, 'Theorems on Fourier series and power series. II', Proc. London Math. Soc. 42 (1936), 52-89.

[20] D. H. Luecking, 'A new proof of an inequality of Littlewood and Paley', Proc. Amer. Math. Soc. 103 (1988), 887-893.

[21] M. Mateljevic and M. Pavlovic, ' $L^{p}$-behaviour of power series with positive coefficients and Hardy spaces', Proc. Amer. Math. Soc. 87 (1983), 309-316. 
[22] J. Miao, 'A property of analytic functions with Hadamard gaps', Bull. Austral. Math. Soc. 45 (1992), 105-112.

[23] R. Nevanlinna, Analytic functions (Springer, New York, 1970).

[24] G. Piranian, 'Bounded functions with large circular variation', Proc. Amer. Math. Soc. 19 (1968), 1255-1257.

[25] Ch. Pommerenke, 'Über die Mittelwerte und Koeffizienten multivalenter Funktionen', Math. Ann. 145 (1962), 285-296.

[26] - Univalent functions (Vandenhoeck und Ruprecht, Göttingen, 1975).

[27] R. Rochberg and Z. J. Wu, 'Toeplitz operators on Dirichlet spaces', Integral Equations Operator Theory 15 (1992), 57-75.

[28] - 'A new characterization of Dirichlet type spaces and applications', lllinois J. Math. 37 (1993), 101-122.

[29] W. Rudin, 'The radial variation of analytic functions', Duke Math. J. 22 (1955), 235-242.

[30] D. A. Stegenga, 'Multipliers of the Dirichlet spaces', Illinois J. Math. 24 (1980), 113-139.

[31] M. Tsuji, Potential theory in modern function theory (Chelsea Publ. Co.. New York, 1975).

[32] D. C. Ullrich, 'Khinchin's inequality and the zeroes of Bloch functions', Duke Math. J. 57 (1988), 519-535.

[33] I. E. Verbitskii, 'Inner function as multipliers of the space $\mathscr{C}_{\alpha}$ ', Funktsional. Anal. $i$ Prilozhen. 16 (1982), 47-48 (in Russian).

[34] S. A. Vinogradov, 'Multiplication and division in the space of analytic functions with areaintegrable derivative, and in some related spaces', Zap. Nauchn. Sem. S.-Peterburg. Otdel. Mat. Inst. Steklov. (POMI) (Issled. po Linein. Oper. i Teor. Funktsii 23) 222 (1995), 45-77, 308 (in Russian); translation in J. Math. Sci. (New York) 87 (1997), 3806-3827.

[35] M. Weiss, 'The law of the iterated logarithm for lacunary trigonometric series', Trans. Amer. Math. Soc. 91 (1959), 444-469.

[36] Z. Wu, 'Carleson measures and multipliers for Dirichlet spaces', J. Funct. Anal. 169 (1999), $148-163$.

[37] A. Zygmund, 'On certain integrals', Trans. Amer. Math. Soc. 55 (1944), 170-204.

[38] - Trigonometric series, Vol. I and Vol. II, 2nd edition (Cambridge Univ. Press, Cambridge, 1959).

Depto. de Análisis Matemático

Facultad de Ciencias

Universidad de Málaga

Campus de Teatinos

29071 Málaga

Spain

e-mail: girela@uma.es, pelaez@anamat.cie.uma.es 